\title{
Measurements of Single and Double Spin Asymmetry in $p p$ Elastic Scattering in the CNI Region with Polarized Hydrogen Gas Jet Target
}

\author{
H. Okada*,†, I. Alekseev**, A. Bravar ${ }^{\ddagger}$, G. Bunce ${ }^{\S, \pi}$, S. Dhawan", \\ K.O. Eyser ${ }^{\dagger \dagger}$, R. Gill ${ }^{\S}$, W. Haeberli ${ }^{\ddagger}$, H. Huang ${ }^{\S}$, O. Jinnouchi ${ }^{\S \S}$,

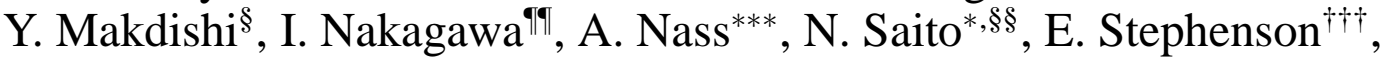 \\ D. Sviridia** ${ }^{* *}$ T. Wise ${ }^{\ddagger}$, J. Wood ${ }^{\S}$ and A. Zelenski ${ }^{\S}$ \\ ${ }^{*}$ Kyoto University, Kyoto Japan \\ ${ }^{\dagger}$ RIKEN, Wako, JAPAN \\ ${ }^{* *}$ Institute for Theoretical and Experimental Physics (ITEP), 117259 Moscow, Russia \\ $¥$ University of Geneva, 1205 Geneva, Switzerland

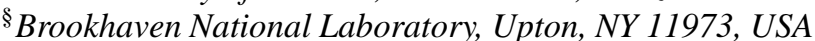 \\ IRIKEN BNL Research Center, Upton, NY 11973, USA \\ Yale University, New Haven, CT 06520, USA \\ ${ }^{\dagger}$ University of California, Riverside, CA 92521, USA \\ \$ University of Wisconsin, Madison, WI 53706, USA

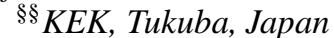 \\ IIII RIKEN, Wako, JAPAN \\ *** University of Erlangen, 91058 Erlangen, Germany \\ ${ }^{\dagger \dagger}$ Indiana University Cyclotron Facility, Bloomington, IN 47408, USA
}

\begin{abstract}
Precise measurements of the single spin asymmetry, $A_{N}$ and the double spin asymmetry, $A_{N N}$, in proton-proton $(p p)$ elastic scattering in the region of four-momentum transfer squared $0.001<-t<0.032(\mathrm{GeV} / c)^{2}$ have been performed using a polarized atomic hydrogen gas jet target and the RHIC polarized proton beam at $24 \mathrm{GeV} / c$ and $100 \mathrm{GeV} / c$. The polarized gaseous proton target allowed us to achieve the measurement of $A_{N N}$ in the CNI region for the first time. Our results of $A_{N}$ and $A_{N N}$ provide significant constraints to determine the magnitude of poorly known hadronic single and double spin-flip amplitudes at this energy.
\end{abstract}

Keywords: Elastic scattering, spin, coulomb nuclear interference

PACS: 13.88.+e, 13.85.Dz, 29.27.Pj, 29.27.Hj

Introduction. $p p$ elastic scattering is one of the most fundamental reactions in particle-nuclear physics and is described in transition amplitudes by use of helicity of initial and final states. Requiring that the interaction is invariant under space inversion, time reversal and rotation in spin space, $p p$ scattering in a given spin state is described in five independent transition amplitudes $\left(\phi_{i}, i=1-5\right)$ as functions of the center-ofmass energy squared, $s$, and $t[1]$. The understanding of these amplitudes would provide crucial guidelines to investigate the reaction mechanism.

Each transition amplitude is described as a sum of the hadronic amplitude $\left(\phi_{i}^{\text {had }}\right)$ and the electro-magnetic amplitude $\left(\phi_{i}^{e m}\right)$. In the small $-t$ region, $\phi_{i}^{e m}$ and $\phi_{i}^{\text {had }}$ become similar in strength and interfere with each other. We call this interference the Coulomb Nuclear Interference (CNI). Thanks to the great successes of QED and the past pre- 

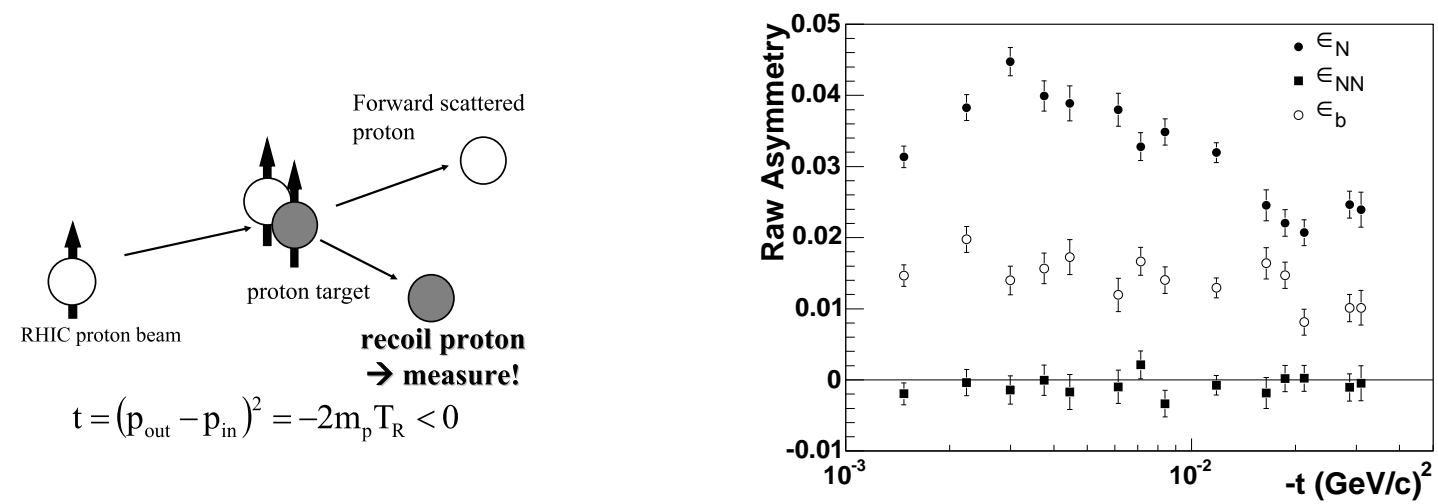

FIGURE 1. Left: Example of "parallel" case, $p^{\uparrow} p^{\uparrow} \rightarrow p p$. Right: $\varepsilon_{N N}, \varepsilon_{N}$ and $\varepsilon_{b}$ at $\sqrt{s}=13.7 \mathrm{GeV}$ as a function of $-t$.

cisely measured quantities (ex. magnetic moment), $\phi_{i}^{e m}$ is precisely described. On the other hand, $\phi_{i}^{\text {had }}$ is not fully described by theory, because the perturbative QCD is not applicable in the CNI region. By use of the experimental data of total and differential cross-section of unpolarized $p p$ elastic scattering, we can determine the sum of two non-spin-flip hadronic amplitudes $\left(\phi_{+}^{\text {had }}=\phi_{1}^{\text {had }}+\phi_{3}^{\text {had }}\right)$ [3]. In order to approach to the hadronic single and double spin-flip amplitudes ( $\phi_{5}^{\text {had }}$ and $\phi_{2}^{\text {had }}$ ), we measure $A_{N}$ and $A_{N N}$ in the CNI region. $A_{N}$ is defined by the asymmetry of cross-section with up-down transverse polarization for one of the protons. Similarly $A_{N N}$ is defined by the asymmetry of cross-section for parallel and anti-parallel transverse polarization for both of the protons. The left side of Fig. 1 depicts "parallel" case. We define the scattering plane from 3-momenta of incident and recoil particles, which is normal to the spin directions. By use of transition amplitudes, $A_{N}$ is expressed as,

$$
A_{N} \approx \frac{-\operatorname{Im}\left[\phi_{5}^{e m}(s, t) \phi_{+}^{h a d *}(s, t)+\phi_{5}^{h a d}(s, t) \phi_{+}^{e m}(s, t)\right]}{\left|\phi_{+}(s, t)\right|^{2}} .
$$

The first term of Eq. 1 is calculable and has a peak around $-t \simeq 0.003(\mathrm{GeV} / c)^{2}$ [2] which is generated by proton's anomalous magnetic moment. Because the presence of $\phi_{5}^{\text {had }}$ introduces a deviation in shape and magnitude from the first term, a measurement of $A_{N}$ in the CNI region, therefore, can be a sensitive probe for $\phi_{5}^{\text {had }}$.

$A_{N N}$ is expressed as,

$$
A_{N N} \approx \frac{2\left|\phi_{5}^{\text {had }}(s, t)\right|^{2}+\operatorname{Re}\left[\left(\phi_{+}(s, t)\right)^{*} \phi_{2}^{\text {had }}(s, t)\right]}{\left|\phi_{+}(s, t)\right|^{2}} .
$$

Because the first term is 2nd order of $\phi_{5}^{\text {had }}$ and the second term is 1 st order of $\phi_{2}^{\text {had }}, A_{N N}$ is sensitive to $\phi_{2}^{\text {had }}$ [4]. From a consequence of angular momentum conservation at small $-t$ and large $\sqrt{s}$, we use $\phi_{4}^{\text {had }} \propto t \rightarrow 0$ for these expressions. 
Experiment. Experiment has been performed using a polarized hydrogen gas jet target and polarized RHIC proton beam at $24 \mathrm{GeV} / c(\sqrt{s}=6.7 \mathrm{GeV})$ and $100 \mathrm{GeV} / c$ $(\sqrt{s}=13.7 \mathrm{GeV})$. We detect the recoil protons by silicon detectors which are located on both sides of the target. The details of experimental setup are described in [5, 6].

In the $p p$ elastic scattering process, both forward-scattered particle and recoil particle are protons and there are no other particles involved nor new particles produced in the process. Since initial states are well defined, the elastic process can be, in principle, identified by detecting the recoil particle only. By measuring kinetic energy $T_{R}$, time of flight, and recoil angle of recoil particle, we measure the mass of recoil particle and all the forward scattered rest particles. We collected 4.3 M events at $\sqrt{s}=13.7 \mathrm{GeV}$ and $0.8 \mathrm{M}$ events at $\sqrt{s}=6.7 \mathrm{GeV}$, respectively. The details of event selection is described in [5].

The selected event yield is sorted by $-t$ bins, which is obtained measuring the kinetic energy of the recoil particle: $-t=2 m_{p} T_{R}$, and spin states. $m_{p}$ is the proton mass. Then we calculate two types of single spin raw asymmetries, $\varepsilon_{N}$ for the target spin state and $\varepsilon_{b}$ for the beam spin state. We also calculate double spin raw asymmetry, $\varepsilon_{N N}$ for the target and beam spin states.

The right side of Fig. 1 displays these raw asymmetries of $\sqrt{s}=13.7 \mathrm{GeV}$ data as a function of $-t$ in the region $0.001 \leq-t \leq 0.035(\mathrm{GeV} / c)^{2}\left(0.5 \leq T_{R} \leq 17 \mathrm{MeV}\right)$. The polarized gaseous proton target allowed us to achieve the measurement in the CNI region for the first time. In order to cancel out the asymmetries of up-down luminosity, and detector acceptance, we employed so-called "square root formula" for $\varepsilon_{N}$ and $\varepsilon_{b}$ calculation. On the other hand, $\varepsilon_{N N}$ needs to be corrected by the luminosity asymmetry. The target spin flips every 5 minutes and the density of both spin states are the same and stable during the experimental period ( $\sim 90$ hours). The beam intensity, which is measured by the wall current monitor [7], varies by bunch (every $106 \mathrm{nsec}$ in 2004) and fill (every several hours). By accumulating intensity for the experimental period, the variation is compensated. Therefore the luminosity asymmetry is quite small compared to statistical error of $\varepsilon_{N N}$.

Results and discussions. $\quad A_{N}$ is measured normalizing $\varepsilon_{N}$ by well measured the target polarization $P_{t}$ [6],

$$
A_{N}=\frac{\varepsilon_{N}}{P_{t}}
$$

Utilizing the measured $A_{N}$, we also measure the beam polarization, $P_{b}=\varepsilon_{b} / A_{N} 1$. Normalizing $\varepsilon_{N N}$ by $P_{t}$ and $P_{b}, A_{N N}$ is obtained via

$$
A_{N N}=\frac{\varepsilon_{N N}}{P_{b} P_{t}}
$$

The left and right plots of Fig. 2 display the results of $A_{N}$ and $A_{N N}$ at $\sqrt{s}=6.7 \mathrm{GeV}$ with filled circles and $13.7 \mathrm{GeV}$ with open circles, respectively. The errors on the data

\footnotetext{
${ }^{1}$ This experimental setup also plays an important role in the RHIC spin program to measure the absolute beam polarization [8].
} 

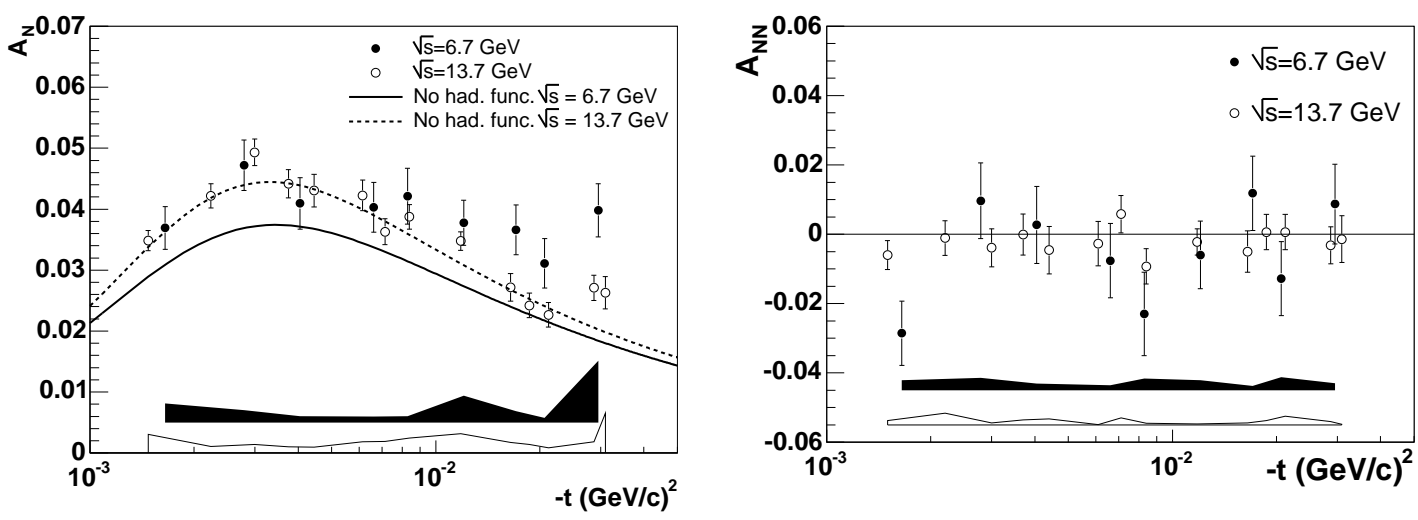

FIGURE 2. The left and right plots display the results of $A_{N}$ and $A_{N N}$ at $\sqrt{s}=6.7 \mathrm{GeV}$ with filled circles and $13.7 \mathrm{GeV}$ with open circles, respectively. The errors on the data points are statistical. The lower bands represents the total systematic errors. The solid and dashed lines in the left plots correspond to the first term in Eq. 1 for these $\sqrt{s}$, respectively.

points are statistical. The lower bands represent the total systematic errors. The solid and dashed lines correspond to the first term in Eq. 1 for these $\sqrt{s}$, respectively.

$A_{N}$ at $\sqrt{s}=13.7 \mathrm{GeV}$ are consistent with the dashed line $\left(\chi^{2} / \mathrm{ndf}=13.4 / 14\right)$. On the other hand, although the accuracy is statistically limited, $A_{N}$ at $\sqrt{s}=6.7 \mathrm{GeV}$ are not consistent with the solid line $\left(\chi^{2} / \mathrm{ndf}=35.5 / 9\right)$ and this discrepancy implies the presence of $\phi_{5}^{\text {had }}$. $A_{N N}$ for these $\sqrt{s}$ have no clear $-t$ dependence and the average values are consistent with zero within $1.5 \sigma$.

In summary, measurements of $A_{N}$ and $A_{N N}$ provide experimental knowledge to poorly known $\phi_{5}^{\text {had }}$ and $\phi_{2}^{\text {had }}$. The $\sqrt{s}$ dependence of $\phi_{5}^{\text {had }}$ is provided by $A_{N}$ results and the theoretical interpretation is under way [9]. However, there is no comprehensive understanding of $\phi_{2}^{\text {had }}$ and $\phi_{5}^{\text {had }}$ yet. Further measurements at different $\sqrt{s}$ are required to fully describe the behavior of $\phi_{2}^{\text {had }}$ and $\phi_{5}^{\text {had }}$.

\section{REFERENCES}

1. M. Jacob and G.C. Wick, Annals Phys. 7, 404 (1959).

2. N.H. Buttimore et al., Phys. Rev. D 59, 114010 (1999).

3. M.M. Block and R.N. Cahn, Czech. J. Phys. 40, 164-175 (1990).

4. T.L. Trueman, RHIC Spin Note, September 27, (2005), hep-ph/0604153

5. H. Okada et al., Phys. Lett. B 638, 450 (2006); H. Okada, Doctororal thesis, July (2006); http://www.star.bnl.gov/ hiromi/HiromiOkadaThesis.pdf

6. A. Zelenski et al., Nucl. Inst. and Meth. A 536, 248 (2005);

T. Wise et al., Nucl. Inst. and Meth. A 559, 1 (2006).

7. P.R. Cameron et al., Nucl.Instrum.Meth. A345, 226-229 (1994).

8. K.O. Eyser, these proceedings (2006).

9. Private discussion with L. Trueman. 